\title{
OBSERVATIONAL EVIDENCE BY FOURIER TRANSFORM SPECTROSCOPY OF CONVECTIVE MOTION IN LATE-TYPE STELLAR ATMOSPHERES
}

\author{
Jean-Pierre Maillard \\ Institut d'astrophysique de Paris \\ 98bis B7v. Arago \\ 75014 Paris \\ France
}

\author{
Daniel Nadeau \\ Département de physique \\ Université de Montréal \\ CP 6128A, Montréal, QC \\ Canada H3C $3 \mathrm{~J} 7$
}

\begin{abstract}
We have obtained FTS spectra of 11 stars of spectral types from MO to M4 III in the infrared $K$ band. The shifts at line bottom of the lines of $\mathrm{CO}$ and $\mathrm{Fe} I$ show a dependence on excitation energy and line depth similar to that caused by convective motions in the photosphere of the Sun but with a larger amplitude. Time variability is small but important differences appear between individual stars of similar types. From these data it should be possible to obtain quantitative estimates of the convective velocities and horizontal temperature inhomogeneities.
\end{abstract}

\section{INTRODUCTION}

The determination of the parameters of convective motions (gas velocities, horizontal temperature inhomogeneities granulation scale) is of considerable interest for the study of red giants as these motions may be related to the isotopic enrichment of the atmosphere, to chromospheric excitation, and to the onset of large mass-loss. For stars far from the solar type, the hydrodynamical calculations are hampered by the lack of suitable initial conditions necessary to follow the evolution of the system towards equilibrium.

To find observational clues to motions in cool stellar atmospheres that could be linked to the parameters of convection, we measure the small differential shifts between the line positions of $\mathrm{CO}$ and metals (principally iron) as a function of excitation energy, line depth, and wavelength; we look for a dependence on spectral type and for differences among stars of the same type, and we monitor stars for time variations.

\section{METHOD}

Convective motions can be measured in the asymmetry and shift of spectral lines (Dravins 1982, Gray 1982). The severe blending of lines in the spectra of late-type stars implies that it is difficult to measure the asymmetry of the profiles. The shift at line bottom is less affected by blending than higher up in the line. Its measurement does not require 
as high a spectral resolution as the measurement of line asymetries so that it is possible to obtain data on a larger sample of stars. By measuring the differential shift of many lines $(\geqslant 100)$, it is possible to obtain its dependence on excitation energy and line depth. Fourier Transform Spectrometry is well suited for this task because of its large spectral coverage and high internal accuracy.

The $\Delta v=2$ and $\Delta v=3$ bands of $C O$ and numerous lines of Fe I can be observed in the infrared $K(2.2 \mu \mathrm{m})$ and $H(1.6 \mu \mathrm{m})$ bands, and cover a large range of excitation energy and line depth. While the absorption in the fundamental band of CO may come from a circumstellar envelope, the overtone bands are almost certainly of photospheric origin. The laboratory frequencies of the near-infrared lines of $\mathrm{CO}$ and $\mathrm{Fe} I$ are almost unique in having an absolute accuracy of $0.001 \mathrm{~cm}^{-1}$ with respect to a common frequency standard and being essentially free of pressure effects (Nadeau and Maillard 1988).

The data of Biemont et a1. (1985) on the infrared spectrum of iron have made it possible to determine the shift of the Fe I lines in the Sun as a function of line depth and excitation energy with an accuracy of $30 \mathrm{~m} \mathrm{~s}^{-1}$ (Nadeau 1988). The solar lines of CO have velocities in agreement with this relation within the $0.001 \mathrm{~cm}^{-1}$ uncertainty.

\section{DIFFERENTIAL LINE SHIFTS IN RED GIANTS}

K-band spectra of 11 giants with spectral types from M0 to M4 III were obtained with the CFHT FTS spectrometer at a resolution of $5 \mathrm{~km} \mathrm{~s}^{-1}$. The stars are selected on the basis of their brightness and their low variability, and double stars are avoided.

The stars HR 0045 and HR 3950 were observed twice, at 23 and 16 month interval respectively. For each of these observations we have plotted in Figure 1 the shifts between the average velocity of the lines of $\mathrm{CO}$ with excitation energy below $1 \mathrm{eV}$, the 1 ines of $\mathrm{CO}$ with energy above $1 \mathrm{eV}$ and the lines of Fe I with average energy near $5.5 \mathrm{eV}$. The reproducibility of the data obtained at different epochs is excellent. It gives an idea of the accuracy of the method and shows that there is little variation of the differential shifts on a time scale of a few years. On the other hand these two stars of similar spectral types have very different relative shifts. There is a large shift between the high and low energy lines of CO in HR 0045 but essentially none in HR 3950.

The data plotted in Figure 2 represent the same differential shifts as in Figure 1 but for all observed stars. A plot of the quantitative relation obtained from the Fe I lines in the Sun, evaluated at average energies and line depths corresponding to the giants data, is shown for comparison. Most red giants show a blueshift of the high excitation energy lines larger than in the Sun, indicating larger convective velocities or stronger temperature inhomogeneities. Some trends do appear as a function of spectral type but there are important individual variations. There is a correlation between the excitation energy and line depth of the observed lines so that it is not straightforward to separate their individual effects. The ratio of the drop in line depth to the increase in energy is high between the low and high energy lines of 
$\mathrm{CO}$, but much smaller between the high energy lines of $\mathrm{CO}$ and the lines of Fe I. One can deduce from this relation that the blueshifts appear more sensitive to excitation energy than line depth for earlier types while the opposite seems true for later types among the observed stars.

From these data we intend to get quantitative estimates of the convective velocities and horizontal temperature inhomogeneities in the photospheres of the red giants. We are also looking for the origin of the large differences between individual stars, which may indicate that additional parameters have an influence on the observed motions.

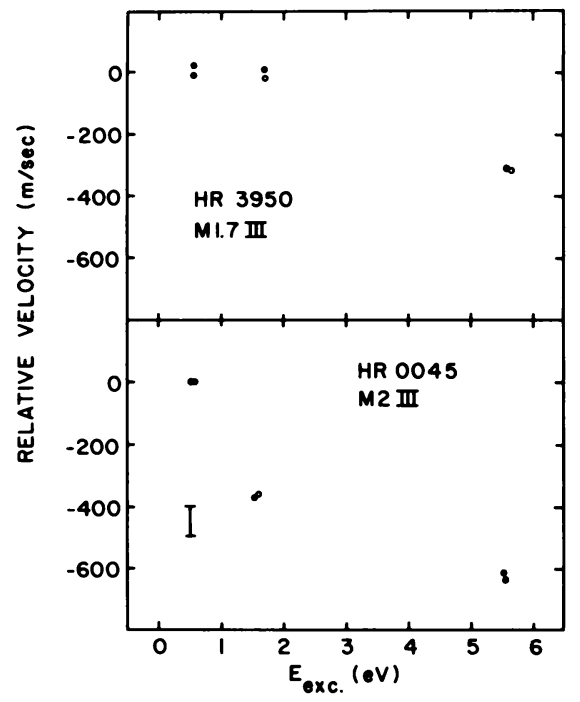

Figure 1. Differential shifts of the lines of $\mathrm{CO}$ and Fe I observed at different epochs. The error bar shows the uncertainty of the laboratory frequencies of $\mathrm{Fe} \mathrm{I}$ with respect to $\mathrm{CO}$.

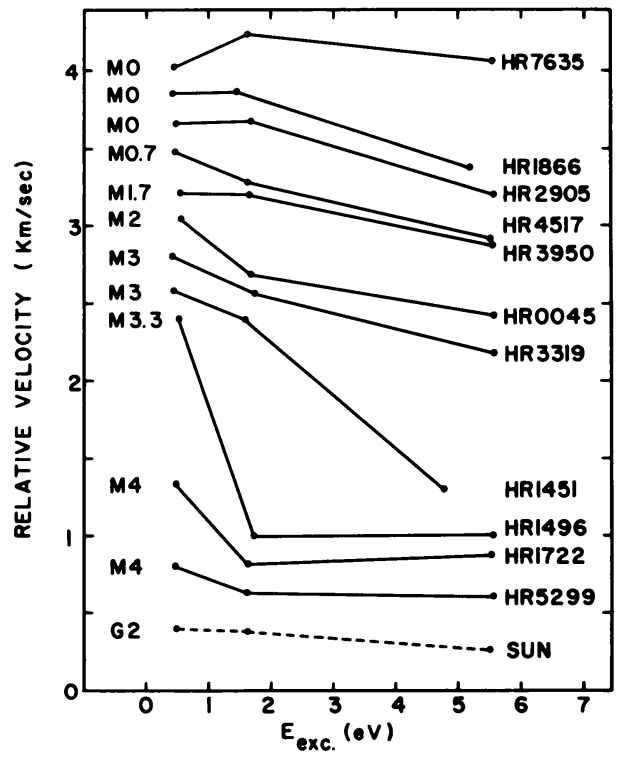

Figure 2. Differential shifts of the lines of $\mathrm{CO}$ and $\mathrm{Fe} I$ for the observed stars. The velocity scale refers to the amplitude of the relative shifts between lines of different energies.

\section{REFERENCES}

Biêmont, E., Brault, J. W., Delbouille, L., and Roland, G. 1985, Astr. Ap. Supp1., 61, 107.

Dravins, D. 1982, Ann. Rev. Astr. Ap., 20, 61.

Gray, D. F. 1982, Ap. J., 255, 200.

Nadeau, D. 1988, Ap. J., 325, in press.

Nadeau, D., and Maillard, J.-P. 1988, Ap. J., submitted. 


\section{DISCUSSION}

RUTTEN: Are there any Fe II lines in your data? Even if they are very weak, it may be valuable to include them. In the theoretical simulations, the ionization equilibrium of iron plays a very important role by influencing the near-ultraviolet and blue line haze, to which the computed granules are quite sensitive. Direct observational comparisons between Fe I and Fe II will be very interesting when comparisons with simulations will be made.

NADEAU: We have not looked for the lines of Fe II because their laboratory frequencies are not known to high accuracy. There may also be a chromospheric contribution to these lines that would make the interpretation of the shifts at line bottom ambiguous. But we can certainly measure them if the lines appear in our spectra, and it is possible that improved laboratory data will be available in the near future. 